\title{
THE CONTINUOUS DIOPHANTINE APPROXIMATION MAPPING OF SZEKERES
}

\author{
JEFFREY C. LAGARIAS and ANDREW D. POLLINGTON
}

(Received 4 January 1993; revised 5 October 1994)

Communicated by J. H. Loxton

To George Szekeres on his $84^{\text {th }}$ birthday

\begin{abstract}
Szekeres defined a continuous analogue of the additive ordinary continued fraction expansion, which iterates a map $T$ on a domain which can be identified with the unit square $[0,1]^{2}$. Associated to it are continuous analogues of the Lagrange and Markoff spectrum. Our main result is that these are identical with the usual Lagrange and Markoff spectra, respectively; thus providing an alternative characterization of them.

Szekeres also described a multi-dimensional analogue of $T$, which iterates a map $T_{d}$ on a higherdimensional domain; he proposed using it to bound $d$-dimensional Diophantine approximation constants. We formulate several open problems concerning the Diophantine approximation properties of the map $T_{d}$.

1991 Mathematics subject classification (Amer. Math. Soc.): primary 11J06, 11J70; secondary 58F03, $58 \mathrm{~F} 08$.
\end{abstract}

\section{Introduction}

Szekeres [14] constructed a continuous analogue of the additive ordinary continued fraction algorithm, which proceeds by iterating a map $T$ from a two-dimensional space of 'configurations' to itself. This space can be naturally identified with the unit square $[0,1]^{2}$. He also defined a multi-dimensional generalization, which iterates a map $T_{d}$ on a certain multi-dimensional space of 'configurations.' His motivation was to use these continuous analogues to obtain bounds for Diophantine approximation constants in dimensions $d \geq 2$, as described in Section 6 .

(C) 1995 Australian Mathematical Society $0263-6115 / 95 \$ A 2.00+0.00$ 
The main purpose of this paper is to study the one-dimensional Szekeres map $T$ in detail. For this map one can define in a natural way an analogue of the Lagrange spectrum in one-dimensional Diophantine approximation; we call this the Szekeres $L$-spectrum. Recall that the one-dimensional approximation constant of $\theta \in \mathbb{R}$ is

$$
C_{1}(\theta)=\liminf _{q \rightarrow \infty} q\{\{q \theta\}\}
$$

where $\{\{x\}\}$ denotes the distance of $x$ to the nearest integer, and that the Lagrange spectrum $\mathscr{L}$ is defined by

$$
\mathscr{L}=\left\{C_{1}(\theta)^{-1}: C_{1}(\theta)>0\right\} .
$$

The Szekeres $L$-spectrum $\mathscr{L}_{S}$ is defined in Section 2, and automatically satisfies $\mathscr{L} \subseteq \mathscr{L}_{s}$. In Section 3 we prove that the Szekeres $L$-spectrum coincides with the Lagrange spectrum. The proof involves a study of the symbolic dynamics attached to the map $T$, and, as an aid in this study, in Section 4 we characterize all points in $[0,1]^{2}$ having the same forward symbolic dynamics under $T$.

In Section 5 we obtain analogous results for the Markoff spectrum of indefinite binary quadratic forms. We show that there is a natural description of the Markoff spectrum in terms of the Szekeres map $T$ (Theorem 5.1). The proof of this result constructs a conjugacy of the Szekeres map to a map encoding an additive variant of the reduction theory of indefinite binary quadratic forms.

A consequence of these results is that the Szekeres map $T$ provides a natural discrete framework in which to study the Lagrange and Markoff spectra. Basic results on the Lagrange and Markoff spectra can be found in Cusick and Flahive [6].

In the final Section 6 we turn to the multi-dimensional Szekeres map $T_{d}$, and state a number of open problems concerning approximation constants associated to this map.

\section{One-dimensional Szekeres map}

The Szekeres map is constructed by analogy with the additive form of the onedimensional continued fraction algorithm. In that algorithm, the $n$th stage of approximating $\xi \in[0,1]$, specifies that $\xi$ is contained in a particular Farey interval $I_{n}=\left[p / q, p^{\prime} / q^{\prime}\right]$ starting from the initial interval $I_{0}=[0 / 1,1 / 1]$. A Farey interval is an interval $\left[p / q, p^{\prime} / q^{\prime}\right]$ such that $p^{\prime} / q^{\prime}-p / q=1 / q q^{\prime}$. The interval $I_{n}$ is split into two subintervals at its mediant $p+p^{\prime} / q+q^{\prime}$, and the $(n+1)$-st stage $I_{n+1}$ chooses the subinterval containing $\xi$. (There are two choices if $\xi=p+p^{\prime} / q+q^{\prime}$.) At each stage a new endpoint $r_{n+1} / s_{n+1}=\left(p+p^{\prime}\right) /\left(q+q^{\prime}\right)$ is added, and one of the two endpoints of $I_{n}$ is dropped. The endpoint $r_{n+1} / s_{n+1}$ is called the $(n+1)$-st additive continued fraction convergent. These additive convergents are precisely the 
set of convergents and intermediate convergents of the ordinary continued fraction algorithm (see Richards [12]).

Szekeres' idea is to introduce a continuous parameter $w \geq 1$ called the weight, and then to do a similar process starting instead with the interval $\tilde{I}_{0}=[0 / w, 1 / 1]$. At the $n$th stage $\xi$ is contained in an interval $\tilde{I}_{n}=[a /(a+b w), c /(c+d w)]$ where $\left[\begin{array}{ll}a & b \\ c & d\end{array}\right] \in S L(2, \mathbb{Z})$. This interval is split at its weighted mediant

$$
(a+c) /(a+c+(b+d) w)
$$

and the $(n+1)$-st interval $\tilde{I}_{n+1}$ is taken to be the subinterval containing $\xi$. Call this procedure the un-normalized Szekeres algorithm. The length of the $n$th interval is:

$$
\left|\tilde{I}_{n}\right|=w /((a+b w)(c+d w)) .
$$

Thus $\left|\tilde{I}_{n}\right| \rightarrow 0$ as $n \rightarrow \infty$, since $a+b+c+d \rightarrow \infty$, and the nested intervals $\tilde{I}_{n}$ converge to $\{\xi\}$.

We obtain the Szekeres map $T$ by a renormalization of this procedure, that identifies the interval with $[0,1]$ at each step, and also keeps track of a rescaled weight. Szekeres calls the resulting space $(\xi, w) \in[0,1] \times[1, \infty)$ the space of normalized configurations $\mathscr{C}$. The initial interval $\tilde{I}_{0}$ has weight $w$ assigned to its left endpoint 0 and weight 1 assigned to its right endpoint 1 . The weighted mediant $1 /(w+1)$ (which always has $1 /(w+1) \leq 1 / 2$ ) is assigned the weight $w+1$. After the interval is split, the chosen subinterval is affinely rescaled to have length 1 , then a reflection $x \rightarrow 1-x$ is used if necessary to move the endpoint with larger weight to 0 . The endpoint weights, which are now either $(w+1,1)$ or $(w+1, w)$, are rescaled to make the weight at 1 equal to 1 , so the resulting new weight $w^{\prime}$ at the endpoint 0 is:

$$
w^{\prime}= \begin{cases}\frac{w+1}{w} & \text { if } 0 \leq \xi \leq \frac{1}{w+1} \\ w+1 & \text { if } \frac{1}{w+1}<\xi \leq \frac{1}{w+1} .\end{cases}
$$

The point $\xi^{\prime}$ specifies the location of $\xi$ in the renormalized subinterval, and the resulting Szekeres map $T: \mathscr{C} \rightarrow \mathscr{C}$ is:

$$
T(\xi, w)= \begin{cases}\text { (a) }\left(1-(w+1) \xi, \frac{w+1}{w}\right) & \text { if } 0 \leq \xi \leq \frac{1}{w+1}, \\ \text { (b) }\left(\left(\frac{w+1}{w}\right)(\xi-1)+1, w+1\right) & \text { if } \frac{1}{w+1}<\xi \leq \frac{1}{w+1} .\end{cases}
$$

In this definition the ambiguity arising in the splitting operation when $\xi=1 /(w+1)$ is resolved by always choosing the left interval. 
Szekeres assigns to each normalized configuration $(\xi, w)$ an approximation number

$$
\Gamma(\xi, w)=\xi w,
$$

which plays the role of a Diophantine approximation measure.

Now set $\left(\xi_{n}, w_{n}\right)=T^{(n)}\left(\xi_{0}, w_{0}\right)$. It is easy to check that, if $w_{0}=1$, we recover the additive continued fraction algorithm, and the weight $w_{n}$ then encodes information about the denominators of the $n$th Farey interval $I_{n}=\left[p / q, p^{\prime} / q^{\prime} /\right]$, namely,

$$
w_{n}= \begin{cases}q / q^{\prime} & \text { if } q \geq q^{\prime}, \\ q^{\prime} / q & \text { if } q^{\prime}>q .\end{cases}
$$

Furthermore an easy calculation shows that the approximation number $\Gamma\left(\xi_{n}, w_{n}\right)$ is just the Diophantine approximation error

$$
\Gamma\left(\xi_{n}, w_{n}\right)=q_{n}\left|q_{n} \xi-p_{n}\right|,
$$

where $q_{n}$ is the $n$th additive continued fraction convergent. (Here $q_{n}$ is $q$ or $q^{\prime}$, whichever is larger.).

We define for a configuration $(\xi, w)$ the Szekeres continuous Diophantine approximation constant $\Gamma^{*}(\xi, w)$ by

$$
\begin{aligned}
\Gamma^{*}(\xi, w) & =\liminf _{n \rightarrow \infty} \Gamma\left(T^{(n)}(\xi, w)\right) \\
& =\liminf _{n \rightarrow \infty} \xi_{n} w_{n} .
\end{aligned}
$$

The Szekeres L-spectrum is

$$
\mathscr{L}_{S}:=\left\{\frac{1}{\Gamma^{*}(\xi, w)}:(\xi, w) \in \mathscr{C} \text { and } \Gamma^{*}(\xi, w)>0\right\}
$$

By the preceding remarks

$$
\Gamma^{*}(\theta, 1)=C_{1}(\theta),
$$

the one-dimensional approximation constant of $\theta$, and this shows that the Szekeres $L$-spectrum contains the Lagrange spectrum:

$$
\mathscr{L} \subseteq \mathscr{L}_{S}
$$

The extra flexibility introduced by allowing continuous variables for the weights $w$, rather than discrete ones, allows the possibility that the Szekeres $L$-spectrum might be strictly larger than the Lagrange spectrum. However, in Section 3 we show they are equal.

An important feature of the Szekeres map $T$ is that it is one-to-one off a set of Lebesgue measure zero. To see this, set $T(\xi, w)=\left(\xi^{\prime}, w^{\prime}\right)$. If case (2.2a) occurs then 
$1<w^{\prime} \leq 2$, while if $(2.2 \mathrm{~b})$ occurs then $w^{\prime} \geq 2$. Consequently the map $T$ is uniquely invertible off the measure zero subset where $w^{\prime}=1$ or 2 , and its inverse function is:

$$
T^{-1}\left(\xi^{\prime}, w^{\prime}\right)= \begin{cases}\text { (a) }\left(\left(\frac{w^{\prime}-1}{w^{\prime}}\right)\left(1-\xi^{\prime}\right), \frac{1}{w^{\prime}-1}\right) & \text { if } 1<w^{\prime}<2, \\ \text { (b) }\left(\left(\frac{w^{\prime}-1}{w^{\prime}}\right) \xi^{\prime}+\frac{1}{w^{\prime}}, w^{\prime}-1\right) & \text { if } w^{\prime} \geq 2 .\end{cases}
$$

By restricting the domain of $T$ slightly we make it a bijection. To do this note that points with $w^{\prime}=2$ have two preimages, both having $w=1$, while points with $w^{\prime}=1$ have no preimages. Thus the configuration space naturally partitions into two pieces $\mathscr{C}_{0} \cup \mathscr{C}_{\infty}$, where $\mathscr{C}_{0}$ consists of all $(\xi, w)$ that have some preimage under $T$ with $w=1$; these are exactly all forward orbits of the points $\{(\xi, 1): 0 \leq \xi \leq 1\}$. The set $\mathscr{C}_{\infty}$ consists of all other points. We call the points in $\mathscr{C}_{0}$ dependent points; the points in $\mathscr{C}_{\infty}$ are independent points. $T$ is a bijection on the set $\mathscr{C}_{\infty}$ of independent points. The set of dependent points $\mathscr{C}_{0}$ has two-dimensional Lebesgue measure zero.

Finally, we note that the configuration space $\mathscr{C}$ can be naturally identified with a measure one subset of $[0,1]^{2}$, by replacing $w$ with a new variable representing a point in a 1-simplex $\Delta_{1}^{*}$ having barycentric coordinates $(w / w+1,1 / w+1)$; see Appendix A.

\section{Lagrange spectrum}

We prove the following result.

THEOREM 3.1. The Szekeres L-spectrum equals the Lagrange spectrum, that is,

$$
\mathscr{L}_{S}=\mathscr{L}
$$

PROOF. We already observed that $\mathscr{L} \subseteq \mathscr{L}_{S}$, so it suffices to show that $\mathscr{L}_{S} \subseteq \mathscr{L}$. Suppose that $(\xi, w) \in \mathscr{C}$ is given, and set

$$
\left(\xi_{n}, w_{n}\right)=T^{(n)}(\xi, w) .
$$

We keep track of the iterates of $(\xi, w)$ using a symbolic dynamics associated to $T$, which specifies which of the mappings

$$
\begin{aligned}
& \mathrm{L}(\xi, w)=(1-(w+1) \xi,(w+1) / w) \\
& \mathrm{R}(\xi, w)=((w+1 / w) \xi-1 / w, w+1)
\end{aligned}
$$

is applied at each step. The itinerary of $(\xi, w)$ is described by an infinite word

$$
\mathrm{W}=S_{1} S_{2} S_{3} \ldots
$$


in the alphabet $\{L, R\}$, with each $S_{i}=L$ or $R$. Now given any infinite word $W$ in $\{L, R\}$ there exists some $\xi^{\prime} \in[0,1]$ such that $\left(\xi^{\prime}, 1\right) \in \mathscr{C}_{0}$ has itinerary W. This follows because for the initial weight $w_{0}=1$ the map $T$ follows the additive continued fraction algorithm, for which all symbol sequences are legal. In fact if $\xi^{\prime}$ has the ordinary continued fraction expansion

$$
\xi^{\prime}=\left[0,1+a_{1}, 1+a_{2}, 1+a_{3}, \ldots\right],
$$

then the word $W=W\left(\xi^{\prime}, 1\right)$ is given by:

$$
W\left(\xi^{\prime}, 1\right)= \begin{cases}\mathrm{LR}^{a_{1}-1} \mathrm{LR}^{a_{2}} \mathrm{LR}^{a_{3}} \cdots & \text { if } a_{1}>0, \\ \mathrm{R}^{a_{2}+1} \mathrm{LR}^{a_{3}} \mathrm{LR}^{a_{4}} \cdots & \text { if } a_{1}=0 .\end{cases}
$$

A detailed proof of this fact is given in Lemma 4.2 following.

To prove $\mathscr{L}_{S} \subseteq \mathscr{L}$ we will show that

$$
\Gamma^{*}(\xi, w)^{-1}=\Gamma^{*}\left(\xi^{\prime}, 1\right)^{-1} \in \mathscr{L} .
$$

The argument has two cases.

Case $1 . \Gamma^{*}\left(\xi^{\prime}, 1\right)=0$. This case occurs if and only if the continued fraction expansion of $\xi^{\prime}$ has unbounded partial quotients. Thus there exist arbitrarily large $a_{i}$ 's in (3.3). We will derive from this that for any $\epsilon>0$ there exist

$$
\xi_{n} w_{n}<\epsilon,
$$

and this implies $\Gamma^{*}(\xi, w)=0$.

Thus we can find $\left(\xi_{n}, w_{n}\right)$ such that the next symbols in the itinerary are $\mathrm{LR}^{a}$ for arbitrarily large $a$. The effect of $T$ is calculated using

$$
\begin{aligned}
\mathrm{L}(\xi, w) & =\left(1-(w+1) \xi, \frac{w+1}{w}\right):=\left(\xi^{\prime}, w^{\prime}\right), \\
\mathrm{R}^{(m)}\left(\xi^{\prime}, w^{\prime}\right) & =\left(\frac{w^{\prime}+m}{w^{\prime}}\left(\xi^{\prime}-1\right)+1, w^{\prime}+m\right) .
\end{aligned}
$$

Since $1 \leq w^{\prime}<2$, on taking $m=a$ the condition $\left(w^{\prime}+m\right)\left(\xi^{\prime}-1\right) / w^{\prime}+1 \geq 0$ yields $1 \geq \xi^{\prime} \geq 1-2 / a$. Hence $\xi_{n} w_{n}:=\xi w \leq \xi(w+1)=1-\xi^{\prime} \leq 2 / a$. Taking $a$ large enough gives $\xi_{n} w_{n}<\epsilon$, completing Case 1 .

Case 2. $\Gamma^{*}\left(\xi^{\prime}, 1\right)>0$. In this case the continued fraction expansion of $\xi^{\prime}$ has bounded partial quotients, and we may suppose that all $a_{i} \leq K$. Set

$$
\left(\xi_{n}^{\prime}, w_{n}^{\prime}\right)=T^{(n)}\left(\xi^{\prime}, 1\right) .
$$

The partial quotient bound guarantees that

$$
1+\frac{1}{K+1} \leq w_{n}^{\prime} \leq K+1
$$


holds for all $n \geq 2$. To prove (3.5) in this case it suffices to show that

$$
\begin{aligned}
& \left|w_{n}-w_{n}^{\prime}\right| \rightarrow 0 \quad \text { as } \quad n \rightarrow \infty, \\
& \left|\xi_{n}-\xi_{n}^{\prime}\right| \rightarrow 0 \quad \text { as } \quad n \rightarrow \infty,
\end{aligned}
$$

since (3.6) then guarantees that $\left|\xi_{n} w_{n}-\xi_{n}^{\prime} w_{n}^{\prime}\right| \rightarrow 0$ as $n \rightarrow \infty$.

We deal first with (3.7a). Observe that we can keep track of the second coordinate $w_{n}$ using the linear fractional transformations

$$
\bar{L}=\left[\begin{array}{ll}
1 & 1 \\
1 & 0
\end{array}\right], \quad \bar{R}=\left[\begin{array}{ll}
1 & 1 \\
0 & 1
\end{array}\right],
$$

since one has $w_{n}=\overline{\mathrm{S}}_{n} \overline{\mathrm{S}}_{n-1} \ldots \overline{\mathrm{S}}_{1}\left(w_{0}\right)$. Note that $\operatorname{det}(\overline{\mathrm{L}})=-1$, which comes from the inversion $x \rightarrow 1-x$ used in the Szekeres renormalization of the interval. Thus to encode the Szekeres map we use matrices in

$$
G L(2, \mathbb{Z})=\left\{M=\left[\begin{array}{ll}
a & b \\
c & d
\end{array}\right]: M \text { integer and } \operatorname{det}(\mathrm{M})= \pm 1\right\},
$$

rather than $S L(2, \mathbb{Z})$. Now set

$$
\mathrm{U}_{n}:=\overline{\mathrm{S}}_{n} \cdots \overline{\mathrm{S}}_{1}=\left[\begin{array}{ll}
a_{n} & b_{n} \\
c_{n} & d_{n}
\end{array}\right]
$$

and observe that $\operatorname{det}\left(\mathrm{U}_{n}\right)= \pm 1$. Since the word $\mathrm{W}$ does not have more than $K$ consecutive letters the same, and since $\bar{L} \bar{R}=\left[\begin{array}{ll}1 & 2 \\ 1 & 1\end{array}\right]$, it is easy to see that

$$
t_{n}=\min \left(a_{n}, b_{n}, c_{n}, d_{n}\right) \geq 2^{\lfloor n / 2 K\rfloor},
$$

since $t_{n+1} \geq t_{n}$ and $t_{n+2} \geq 2 t_{n}$ whenever an LR occurs. Now

$$
\begin{aligned}
\left|w_{n}-w_{n}^{\prime}\right| & =\left|\mathrm{U}_{n} w_{0}-\mathrm{U}_{n} w_{0}^{\prime}\right| \\
& =\left|\frac{a_{n} w_{0}+b_{n}}{c_{n} w_{0}+d_{n}}-\frac{a_{n} w_{0}^{\prime}+b_{n}}{c_{n} w_{0}^{\prime}+d_{n}}\right| \\
& =\frac{\left|w_{0}-w_{0}^{\prime}\right|}{\left(c_{n} w_{0}+d_{n}\right)\left(c_{n} w_{0}^{\prime}+d_{n}\right)} \\
& \leq\left|w_{0}-w_{0}^{\prime}\right| \frac{1}{d_{n} d_{n}^{\prime}} \leq\left|w_{0}-w_{0}^{\prime}\right| 2^{-2\lfloor n / 2 K\rfloor} .
\end{aligned}
$$

This proves (3.7a)

Next consider $\xi_{n}$ and $\xi_{n}^{\prime}$. If $w_{n}, w_{n}^{\prime}$ are close, then since $\left(\xi_{n}, w_{n}\right),\left(\xi_{n}^{\prime}, w_{n}^{\prime}\right)$ have identical itineraries

$$
W_{n+1}=S_{n+1} S_{n+2} S_{n+3} \cdots,
$$


we can show that this forces $\xi_{n}$ to be close to $\xi_{n}^{\prime}$. Consider the linear fractional transformations and their inverses,

$$
\begin{array}{ll}
\mathrm{L}(w)=\left[\begin{array}{cc}
-(w+1) & 1 \\
0 & 1
\end{array}\right], & \mathrm{L}(w)^{-1}=\left[\begin{array}{cc}
-\frac{1}{w+1} & \frac{1}{w+1} \\
0 & 1
\end{array}\right], \\
\mathrm{R}(w)=\left[\begin{array}{cc}
w+1 & -1 \\
0 & w
\end{array}\right], & \mathrm{R}(w)^{-1}=\left[\begin{array}{cc}
\frac{1}{w+1} & \frac{1}{w(w+1)} \\
0 & \frac{1}{w}
\end{array}\right] .
\end{array}
$$

These depend on the parameter $w$. We encode the action of $T$ on $\xi_{n}$ for $s$ iterations as:

$$
\xi_{n+s}=\mathrm{S}_{n+s}\left(w_{n+s-1}\right) \mathrm{S}_{n+s-1}\left(w_{n+s-2}\right) \cdots \mathrm{S}_{n+1}\left(w_{n}\right) \xi_{n},
$$

Consequently

$$
\xi_{n}=\mathrm{S}_{n+1}\left(w_{n}\right)^{-1} \ldots \mathrm{S}_{n+s}\left(w_{n+s-1}\right)^{-1}\left(\xi_{n+s}\right) .
$$

Note that both $\mathrm{L}(w)^{-1}$ and $\mathrm{R}(w)^{-1}$ are contractions when $w \geq 1$. Since no $K$ consecutive letters are the same, one can show, using the bounded partial quotient condition (3.6), that the contraction ratio in (3.12) is at least $(1+1 /(K+1))^{-\lfloor s / 2 K\rfloor}$. Now take $n_{0}$ so large that $\left|w_{n}-w_{n}^{\prime}\right| \leq \epsilon$ for all $n \geq n_{0}$. We trivially know that $\left|\xi_{n+s}-\xi_{n+s}^{\prime}\right| \leq 1$ and using the contracting property in (3.11) and the fact that $w_{n}$ and $w_{n}^{\prime}$ are close, it follows that

$$
\left|\xi_{n}-\xi_{n}^{\prime}\right| \leq 2^{-\lfloor s / 2 K\rfloor}+(K+2) \epsilon
$$

for all $n \geq n_{0}$. We can take $s$ arbitrarily large and $\epsilon$ arbitrarily small, which proves (3.7b). This completes Case 2 and the proof.

\section{Characterizing the forward symbolic dynamics of $T$}

In this section we will characterize which initial configurations $(\xi, w)$ yield the same word $W$, and derive (3.4). Let $W(\xi, w)$ denote the itinerary of the Szekeres map $T$ on $(\xi, w)$.

LEMMA 4.1. (a) $\mathrm{W}\left(\xi_{1}, w_{1}\right)=\mathrm{W}\left(\xi_{2}, w_{2}\right)$ if and only if

$$
\xi_{1} w_{1} /\left(1-\xi_{1}\right)=\xi_{2} w_{2} /\left(1-\xi_{2}\right) .
$$

In particular, 
(b) $\mathrm{W}(\xi, w)=\mathrm{W}\left(\xi^{\prime}, 1\right)$ if and only if

$$
\xi^{\prime}=\xi w /(1+(w-1) \xi)
$$

ProOF. Consider the Szekeres map for an initial pair $(\xi, w)$ applied without renormalization at each stage. The word $\mathrm{W}$ then induces a sequence $a_{n}, b_{n}$ (as in (2.1)) so that

$$
a_{n} /\left(a_{n}+b_{n} w\right) \rightarrow \xi \quad \text { as } \quad n \rightarrow \infty
$$

But the same word applied to $\left(\xi^{\prime}, 1\right)$ induces the sequence $a_{n}, b_{n}$ again where now

$$
a_{n} /\left(a_{n}+b_{n}\right) \rightarrow \xi^{\prime} \quad \text { as } \quad n \rightarrow \infty
$$

Thus

$$
\lim _{n \rightarrow \infty} b_{n} / a_{n}=1 / \xi^{\prime}-1
$$

and substituting in (4.1) we obtain

$$
1+\left(1 / \xi^{\prime}-1\right) w=1 / \xi
$$

which in turn gives part (b) and then part (a). The converse follows since the word $W$ is uniquely determined by $(\xi, w)$.

LEMMA 4.2. If $\xi^{\prime}$ has the ordinary continued fraction expansion

$$
\xi^{\prime}=\left[0,1+a_{1}, 1+a_{2}, \ldots\right]
$$

with all $a_{i} \geq 0$, then the itinerary of $\left(\xi^{\prime}, 1\right)$ under the Szekeres map $T$ is:

$$
W\left(\xi^{\prime}, 1\right)= \begin{cases}\mathrm{LR}^{a_{1}-1} \mathrm{LR}^{a_{2}} \mathrm{LR}^{a_{3}} \ldots & \text { if } a_{1} \geq 1, \\ \mathrm{R}^{a_{2}+1} \mathrm{LR}^{a_{3}} \mathrm{LR}^{a_{4}} \ldots & \text { if } a_{1}=0 .\end{cases}
$$

PROOF. To facilitate the calculations we introduce a new map $\tau:[0,1] \rightarrow[0,1]$ as follows:

$$
\tau(x)= \begin{cases}\frac{2 x-1}{x-1} & \text { if } 0 \leq x \leq 1 / 2, \quad \text { (L) } \\ \frac{2 x-1}{x} & \text { if } 1 / 2<x \leq 1\end{cases}
$$

Let $W_{\tau}(x)$ denote the itinerary of $x$ under $\tau$. By Lemma 4.1

$$
W(x, 2)=W\left(\frac{2 x}{1+x}, 1\right)
$$


Since

$$
T(x, 1)= \begin{cases}(1-2 x, 2) & \text { if } x \leq 1 / 2 \\ (2 x-1,2) & \text { if } x>1 / 2\end{cases}
$$

we obtain

$$
\begin{aligned}
W(x, 1) & = \begin{cases}\operatorname{LW}(1-2 x, 2) & \text { if } x \leq 1 / 2, \\
\operatorname{RW}(2 x-1,2) & \text { if } x>1 / 2,\end{cases} \\
& = \begin{cases}\operatorname{LW}\left(\frac{1-2 x}{1-x}, 1\right) & \text { if } x \leq 1 / 2, \\
\operatorname{RW}\left(\frac{2 x-1}{x}, 1\right) & \text { if } x>1 / 2 .\end{cases}
\end{aligned}
$$

This shows the connection between $T$ and $\tau$. We have

$$
W(x, 1)=W_{\tau}(x),
$$

where $\tau$ is a fractional linear transformation defined by the matrices

$$
\mathscr{L}=\left(\begin{array}{cc}
2 & -1 \\
1 & -1
\end{array}\right), \quad \mathscr{R}=\left(\begin{array}{cc}
2 & -1 \\
1 & 0
\end{array}\right) .
$$

Let

$$
\mathscr{A}=\left(\begin{array}{cc}
0 & 1 \\
1 & 0
\end{array}\right), \quad \mathscr{B}=\left(\begin{array}{cc}
1 & -1 \\
0 & 1
\end{array}\right)
$$

Then one finds that

$$
\mathscr{L}=\mathscr{A} \mathscr{B}^{-1} \mathscr{A} \mathscr{B}^{2} \mathscr{A}, \quad \mathscr{R}=\mathscr{A} \mathscr{B}^{-1} \mathscr{A} \mathscr{B} \mathscr{A} \mathscr{B} \mathscr{A}
$$

By considering the action of $\mathscr{A}$ and $\mathscr{B}$ on the continued fraction we obtain

$$
\begin{aligned}
\mathrm{W}_{\tau}\left(\left[0,1+a_{1}, 1+a_{2}, \ldots\right]\right) & =\mathrm{LR}^{a_{1}-1} \operatorname{LR}^{a_{2}} \operatorname{LR}^{a_{3}} \cdots \quad \text { if } a_{1}>0 \\
\mathrm{~W}_{\tau}\left(\left[0,1,1+a_{2}, \ldots\right]\right) & =\mathrm{R}^{a_{2}+1} \operatorname{LR}^{a_{3}} \operatorname{LR}^{a_{4}} \ldots
\end{aligned}
$$

This is (4.3).

\section{Markoff spectrum}

The Markoff spectrum is associated to binary quadratic forms

$$
Q(X, Y)=A X^{2}+B X Y+C Y^{2}
$$


which are indefinite, that is, $\operatorname{det}(Q)=B^{2}-4 A C>0$. The Markoff constant $\Gamma_{M}(Q)$ of $Q$ is

$$
\Gamma_{M}(Q)=\operatorname{det}(Q)^{-1 / 2} \inf \left\{|Q(m, n)|:(m, n) \in \mathbb{Z}^{2}-\mathbf{0}\right\} .
$$

The Markoff spectrum is

$$
\mathscr{M}=\left\{\frac{1}{\Gamma_{M}(Q)}: \Gamma_{M}(Q)>0 \text { and } \operatorname{det}(Q)>0\right\} .
$$

Since $\Gamma_{M}(a Q)=\Gamma_{M}(Q)$, there is no loss of generality to consider only forms of determinant 1 . Any such form with $A \neq 0$ factors as

$$
Q(X, Y)=\frac{1}{\rho_{1}+\rho_{2}}\left(X+\rho_{1} Y\right)\left(X-\rho_{2} Y\right)
$$

where $\rho_{1} \geq 0, \rho_{2} \geq 0$, and $\rho_{1}+\rho_{2}>0$. Forms with $A=0$ have Markoff constant $\Gamma_{M}(Q)=0$, and thus play no role in determining the Markoff spectrum.

One can alternatively compute the Markoff constant $\Gamma_{M}(Q)$ using the $G L(2, \mathbb{Z})$ action on forms. If $\mathrm{M}=\left[\begin{array}{ll}a & b \\ c & d\end{array}\right] \in G L(2, \mathbb{Z})$ then

$$
M Q(X, Y):=Q(d X-b Y,-c X+a Y) \text {. }
$$

Call two forms improperly equivalent, written $Q_{1} \approx Q_{2}$, if $Q_{2}=\mathrm{M} Q_{1}$, for some $M \in G L(2, \mathbb{Z})$. The standard theory of equivalence of forms uses an $S L(2, \mathbb{Z})$-action: $Q_{1}$ is properly equivalent to $Q_{2}$ if $M \in S L(2, \mathbb{Z})$. Note that improper equivalence is the natural choice for use with (5.1). Then (5.1) becomes

$$
\Gamma_{M}(Q)=\operatorname{det}(Q)^{-1 / 2} \inf \{|\tilde{Q}(1,0)|: \tilde{Q} \approx Q\}
$$

since for any relatively prime $(m, n)$ there are matrices in $G L(2, \mathbb{Z})$ with $(c, d)=$ $(-n, m)$. The reduction theory of indefinite quadratic forms asserts furthermore that the infimum in the right side of (5.5) remains $\Gamma_{M}(Q)$ when $\tilde{Q}$ is restricted to be a reduced form. A form (5.3) is called reduced if $0 \leq \rho_{1} \leq 1$ and $\rho_{2} \geq 1$, and is called semi-reduced if $0 \leq \rho_{1} \leq 1$ and $\rho_{2} \geq 0$. This definition differs slightly from the usual definition of reduced indefinite form, which is defined for the $S L(2, \mathbb{Z})$-action, and which also calls $\rho_{1} \geq 1$ and $0 \leq \rho_{2} \leq 1$ reduced, cf. Dickson [8]. Note that the matrix $M=\left[\begin{array}{cc}1 & 0 \\ 0 & -1\end{array}\right]$ of determinant -1 takes a reduced form in the latter sense to one in our sense while keeping $|\tilde{Q}(1,0)|$ constant.

The Markoff spectrum has a second definition in terms of geodesics in the hyperbolic plane $\mathbb{W}=\{z: \operatorname{Im}(z)>0\}$. Such geodesics are either semicircles normal to the real axis, specified by their two endpoints in $\mathbb{R}$, or else are vertical lines with 
one endpoint on $\mathbb{R}$ and the other at $i \infty$. We associate to the indefinite form (5.3) the semicircular geodesic $G\left(\rho_{1}, \rho_{2}\right)$ having endpoints at $-\rho_{1}$ and $\rho_{2}$. The exceptional forms with $A=0$ correspond to vertical geodesics with one endpoint at $i \infty$. The height of a semicircular geodesic $G$ is its maximal imaginary part, which is

$$
h t\left(G\left(\rho_{1}, \rho_{2}\right)\right):=h t\left(\rho_{1}, \rho_{2}\right)=\frac{1}{2}\left(\rho_{1}+\rho_{2}\right) .
$$

The Markoff spectrum can be described in terms of heights of geodesics, since

$$
Q(1,0)=\left(\rho_{1}+\rho_{2}\right)^{-1}=\frac{1}{2}\left(h t\left(G\left(\rho_{1}, \rho_{2}\right)\right)\right)^{-1}
$$

The linear fractional action of $\mathrm{M}=\left[\begin{array}{ll}a & b \\ c & d\end{array}\right] \in G L(2, \mathbb{Z})$ sends the geodesic $G\left(\rho_{1}, \rho_{2}\right)$ to

$$
M G\left(\rho_{1}, \rho_{2}\right)=G\left(\frac{a \rho_{1}-b}{-c \rho_{1}+d}, \frac{a \rho_{2}+b}{c \rho_{2}+d}\right) .
$$

This action of $G L(2, \mathbb{Z})$ on quadratic forms and geodesics then gives

$$
\frac{1}{\Gamma_{M}(Q)}=\sup _{M \in G L(2, \mathbb{Z})}\left\{h t\left(\mathrm{M} G\left(\rho_{1}, \rho_{2}\right)\right)\right\} .
$$

The Markoff spectrum has a third definition in terms of a shift on a continued fraction cf. Cusick and Flahive [6]. Let $\left[\ldots, a_{-j}, \ldots, a_{-1}, a_{0}, a_{1}, \ldots, a_{i}, \ldots\right]$ be a doubly infinite sequence of positive integers denoted by $A$. Put

$$
\lambda_{i}(A)=\left[a_{i}, a_{i+1}, \ldots\right]+\left[0, a_{i-1}, a_{i-2}, \ldots\right] .
$$

The Markoff constant $\mathscr{M}(A)$ for $A$ is

$$
\mathscr{M}(A):=\sup _{i \in \mathbb{Z}} \lambda_{i}(A)
$$

The Markoff spectrum is the set of values $\mathscr{M}(A)$ as $A$ varies over all doubly infinite sequences of positive integers. A sequence $A$ corresponds to a quadratic form (5.3) by

$$
\begin{aligned}
& \rho_{1}=\left[0, a_{-1}, a_{-2}, \ldots\right], \\
& \rho_{2}=\left[a_{1}, a_{2}, a_{3}, \ldots\right] .
\end{aligned}
$$

Note that $0<\rho_{1}<1$ and $\rho_{2}>1$, so that these correspond to reduced forms in an essentially one-to-one fashion.

In order to associate a Markoff spectrum to $T$ we wish to associate to each configuration $(\xi, w)$ an indefinite quadratic form. Equivalently we wish to associate an 
oriented geodesic in the upper half-plane $\forall$ to the orbit of $(\xi, w)$. It is natural to expect that the points in $\mathscr{C}_{0}$ should correspond to vertical geodesics having a starting endpoint at $i \infty$, and those in $\mathscr{C}_{\infty}$ to geodesics with both endpoints on the real axis. We treat here only the case $\mathscr{C}_{\infty}$, which corresponds to the Markoff spectrum.

Our object is to prove the following result.

THEOREM 5.1. For a semi-reduced indefinite quadratic form

$$
Q(X, Y)=\frac{1}{\rho_{1}+\rho_{2}}\left(X+\rho_{1} Y\right)\left(X-\rho_{2} Y\right)
$$

with $0<\rho_{1}<1$ and $\rho_{2}>0$ the Markoff constant is given by

$$
\Gamma_{M}(Q)=\inf \left\{w_{k} \xi_{k}: k \in \mathbb{Z}\right\}
$$

where $\left\{\left(\xi_{k}, w_{k}\right): k \in \mathbb{Z}\right\}$ are the forward and backward iterates under the Szekeres map $T$ of $\left(\xi_{0}, w_{0}\right)=\left(\rho_{1} \rho_{2}\left(1+\rho_{1} \rho_{2}\right)^{-1}, \rho_{1}^{-1}\right)$.

The formula (5.9) is in parallel with the Szekeres Lagrange spectrum formula (2.6).

As a preliminary to proving Theorem 5.1 we derive an additive variant of the reduction theory of indefinite quadratic forms. The standard reduction theory of indefinite quadratic forms (cf. Dickson [8, pp. 72-77]) shows that all reduced forms lie in an infinite chain: each reduced form $[A, B, C]$ has a unique left neighbor $[*, *, A]$ and a unique right neighbor $[C, *, *]$ which is reduced. Neighboring forms are found by a process similar to the ordinary continued fraction. To obtain an additive version of this process one must include semi-reduced forms as well. The additive reduction map $U$ takes the roots $\left(\rho_{1}, \rho_{2}\right)$ of a semi-reduced form to that of its semireduced right neighbor. The map $U:[0,1] \times \mathbb{R}^{+} \rightarrow[0,1] \times \mathbb{R}^{+}$is given explicitly by:

$$
U\left(\rho_{1}, \rho_{2}\right)= \begin{cases}\text { (a) }\left(\frac{\rho_{1}}{1-\rho_{1}}, \frac{\rho_{2}}{1+\rho_{2}}\right) & \text { if } 0 \leq \rho_{1} \leq 1 / 2, \\ \text { (b) }\left(\frac{1-\rho_{1}}{\rho_{1}}, \frac{1+\rho_{2}}{\rho_{2}}\right) & \text { if } 1 / 2<\rho_{1} \leq 1 .\end{cases}
$$

On the form level it takes a form $Q$ to its semi-reduced right neighbor $\tilde{Q}$, using the change of variables $(X, Y) \rightarrow(X, Y-X)$ and $(X, Y) \rightarrow(Y, X-Y)$, corresponding to $(5.10 \mathrm{a})$ and $(5.10 \mathrm{~b})$, respectively. (The notion of right-neighbor is not well-defined if $\rho_{1}=1 / 2$.) The map $U$ has the almost-everywhere defined inverse map:

$$
U^{-1}\left(\rho_{1}, \rho_{2}\right)= \begin{cases}\text { (a) }\left(\frac{\rho_{1}}{1+\rho_{1}}, \frac{\rho_{2}}{1-\rho_{2}}\right) & \text { if } 0 \leq \rho_{2}<1, \\ \text { (b) }\left(\frac{1}{1+\rho_{1}}, \frac{1}{\rho_{2}-1}\right) & \text { if } \rho_{2}>1 .\end{cases}
$$


This map takes a quadratic form $Q$ to its semi-reduced left neighbor $\tilde{Q}$, using the changes of variables $(X, Y) \rightarrow(X, X+Y)$ and $(X, Y) \rightarrow(X+Y, X)$, respectively. (The left neighbor is not well-defined if $\rho_{2}=1$.) Set $U^{(n)}\left(\rho_{1}, \rho_{2}\right)=\left(\rho_{1}^{(n)}, \rho_{2}^{(n)}\right)$. The maps $U$ and $U^{-1}$ are one-to-one and onto when restricted to the domain

$$
\tilde{\mathscr{U}}=\left\{\left(\rho_{1}, \rho_{2}\right): \text { for all } n \in \mathbb{Z}, \rho_{1}^{(n)} \neq \frac{1}{2} \text { and } \rho_{2}^{(n)} \neq 1\right\} \text {. }
$$

It is easy to check that any semi-reduced form $Q$ such that $\left(\rho_{1}, \rho_{2}\right) \notin \tilde{\mathscr{U}}$ has $Q(m, n)=$ 0 for some $(m, n) \in \mathbb{Z}^{2}$ with g.c.d. $(m, n)=1$, hence $\Gamma_{M}(Q)=0$, and such forms do not contribute to the Markoff spectrum. If $\rho_{1}=1 / 2$ then $Q(1,-2)=0$ and if $\rho_{2}=1$ then $Q(0,1)=0$.

LEMMA 5.1. Suppose that $Q(X, Y)=\left(\rho_{1}+\rho_{2}\right)^{-1}\left(X+\rho_{1} Y\right)\left(X-\rho_{2} Y\right)$ is semireduced, that is, $0 \leq \rho_{1} \leq 1$ and $\rho_{2} \geq 1$. If $\left(\rho_{1}, \rho_{2}\right) \in \tilde{\mathscr{U}}$ then

$$
\Gamma_{M}(Q)=\inf \left\{\frac{1}{2}\left[h t\left(U^{(k)}\left(\rho_{1}, \rho_{2}\right)\right)\right]^{-1}: k \in \mathbb{Z}\right\} .
$$

PROOF. It suffices to check that the additive reduction map $U$ finds the complete chain of reduced forms equivalent to $Q$. Then one applies the well-known fact that the infimum in (5.5) is achieved over the set of reduced forms. (Recall that reduced forms occur exactly when $\rho_{2}>1$.) We omit the details.

In Appendix $\mathrm{B}$ we show that the additive reduction map $U$ is conjugate to a map $F:[0,1]^{2} \rightarrow[0,1]^{2}$ called the additive map, which has an especially symmetrical form, and which is closely related to the additive one-dimensional continued fraction algorithm.

PROOF OF THEOREM 5.1. We want to explicitly exhibit a conjugating map $\Phi$ that makes the following diagram commute

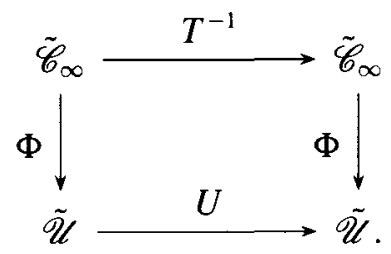

We set $\Phi\left(\xi_{0}, w_{0}\right)=\left(\rho_{1}, \rho_{2}\right)$. To guess what $\Phi$ must be we use the fact that $T^{-1}$ and $U$ both have symbolic dynamics consisting of two-sided shifts on two symbols, except that restricting the domain to $\tilde{\mathscr{C}}_{\infty}$ (respectively $\tilde{\mathscr{U}}$ ) eliminates all symbol sequences having an infinite string of repeated symbols at either end. 
To find the left endpoint $-\rho_{1}$, we first conjugate the inverse map $T^{-1}$ to a map $\tilde{T}^{-1}$ using the change of variable $w=1 / \Psi$. Then $0<\Psi \leq 1$ and we obtain:

(5.15) $\tilde{T}^{-1}(\xi, \Psi):= \begin{cases}\left((1-\Psi) \xi+\Psi, \frac{\Psi}{1-\Psi}\right) & \text { if } 0 \leq \Psi<1 / 2, \\ \left((1-\Psi)(1-\xi), \frac{1-\Psi}{\Psi}\right) & \text { if } 1 / 2 \leq \Psi \leq 1 .\end{cases}$

Comparing this with $U$, we see that both are driven by one coordinate and have identical symbolic dynamics. So we associate to $\left(\xi_{0}, w_{0}\right)$ a geodesic whose left endpoint is $-\Psi$, that is, we take $\rho_{1}\left(\xi_{0}, w_{0}\right)=1 / w_{0}$.

To specify the right endpoint of the geodesic, we associate the point $\rho_{2}=\rho_{2}\left(\xi_{0}, w_{0}\right)$ whose $U^{-1}$-symbolic expansion agrees with that of $\left\{T^{(k)}\left(\xi_{0}, w_{0}\right): k \geq 1\right\}$.

By Lemma 4.1(b) the symbolic expansion $W\left(\xi_{0}, w_{0}\right)=W\left(\xi^{\prime}, 1\right)$, where

$$
\xi^{\prime}=\frac{\xi_{0} w_{0}}{1+\left(w_{0}-1\right) \xi_{0}},
$$

and by (4.7) this in turn equals the symbolic dynamics $W_{\tau}\left(\xi^{\prime}\right)$, where

$$
\tau(x)= \begin{cases}\frac{2 x-1}{x-1} & \text { if } 0 \leq x \leq 1 / 2 \\ \frac{2 x-1}{x} & \text { if } 1 / 2<x \leq 1\end{cases}
$$

Now observe that the symbolic dynamics of $U^{-1}$ is driven by its second coordinate $\rho_{2}$, and under the change of variable $x=1 /\left(\rho_{2}+1\right)$, the map

$$
V\left(\rho_{2}\right)= \begin{cases}\frac{\rho_{2}}{1-\rho_{2}} & \text { if } 0 \leq \rho_{2}<1, \\ \frac{1}{\rho_{2}-1} & \text { if } \rho_{2}>1,\end{cases}
$$

is also conjugated to $\tau(x)$. Since $\rho_{2}=(1-x) / x$, the right endpoint of the desired geodesic should be

$$
\rho_{2}=\frac{1-\xi^{\prime}}{\xi^{\prime}}=\frac{1-\xi_{0}}{\xi_{0} w_{0}} .
$$

The conjugating map $\Phi$ is therefore:

$$
\left(\rho_{1}, \rho_{2}\right)=\Phi\left(\xi_{0}, w_{0}\right):=\left(\frac{1}{w_{0}}, \frac{1-\xi_{0}}{\xi_{0} w_{0}}\right) .
$$

This map is explicitly invertible:

$$
\left(\xi_{0}, w_{0}\right)=\Phi^{-1}\left(\rho_{1}, \rho_{2}\right):=\left(\frac{\rho_{1} \rho_{2}}{1+\rho_{1} \rho_{2}}, \frac{1}{\rho_{1}}\right) .
$$


One can now verify by direct substitution that the diagram (5.14) commutes. The symbolic dynamics guarantees that the domain $\tilde{\mathscr{C}}_{\infty}$ and range $\tilde{\mathscr{U}}$ match.

Finally we observe that under the map $\Phi$ the resulting height is:

$$
h t\left(\rho_{1}, \rho_{2}\right)=\frac{1}{2}\left(\rho_{1}+\rho_{2}\right):=\frac{1}{2}\left(\frac{1}{w_{0}}+\frac{1-\xi_{0}}{\xi_{0} w_{0}}\right)=\frac{1}{2}\left(\frac{1}{\xi_{0} w_{0}}\right) .
$$

This yields (5.9), and completes the proof of Theorem 5.1.

\section{Multi-dimensional approximations}

This section describes open problems concerning the $d$-dimensional Szekeres map $T_{d}$, for $d \geq 2$. The somewhat complicated definition of $T_{d}$ is given in Appendix A. It is constructed as a continuous analogue of the Szekeres [13] multi-dimensional continued fraction.

Szekeres introduced the maps $T_{d}$ as a possible approach to get bounds for supremum norm Diophantine approximation constants. The (sup-norm) approximation constant of $\theta=\left(\theta_{1}, \ldots, \theta_{d}\right) \in \mathbb{R}^{d}$ is

$$
C_{d}(\theta)=\liminf _{q \rightarrow \infty}\left(\max _{1 \leq i \leq d} q\left\{\left\{q \theta_{i}\right\}\right\}^{d}\right),
$$

where $\{\{x\}\}$ is the distance to the nearest integer, and the $d$-dimensional (sup-norm) approximation constant is

$$
C_{d}=\sup _{\theta \in \mathbb{R}^{d}} C_{d}(\theta) .
$$

Szekeres [14] defined a $d$-dimensional continuous approximation constant $S_{d}$ associated to $T_{d}$ (see Appendix A) and showed that it is related to the $d$-dimensional approximation constant by

$$
S_{d} \geq C_{d}
$$

He also wished to use it to study the $d$-dimensional algebraic Diophantine approximation constant $A_{d}$ which is defined similarly to (6.1) except that $\theta$ runs only over vectors for which $\left[1, \theta_{1}, \ldots, \theta_{d}\right]$ is a $\mathbb{Q}$-basis of a real algebraic number field of degree $d+1$, and which obviously satisfies

$$
C_{d} \geq A_{d}
$$

It is well-known that $C_{1}=A_{1}=1 / \sqrt{5}$, and Szekeres [14] announced without proof that

$$
S_{1}=C_{1}=A_{1}=1 / \sqrt{5}
$$


This result now follows from Theorem 3.1 in Section 3. In the two-dimensional case Cassels [3] proved that $A_{2} \geq 2 / 7$ and Adams [1] later showed that $A_{2}=2 / 7$. Szekeres [15] presents numerical evidence supporting the conjecture that in fact

$$
S_{2}=C_{2}=A_{2}=2 / 7 \text {. }
$$

Subsequently Szekeres [16] went on to study the three-dimensional case by numerical experiments. These experiments suggested that $S_{3}>A_{3}$, and also that 'extremal' configurations for the three-dimensional continuous algorithm which are near to attaining the constant $S_{3}$ do not consist of algebraic numbers from a fixed number field. It is not known, however, if $S_{d}$ is finite for any $d \geq 2$.

The first open problem is: For which dimensions $d$ is $S_{d}$ finite? This problem arises in studying the Diophantine approximation properties of the multi-dimensional continued fraction algorithm of Szekeres [13]. Szekeres expressed the hope that his $d$ dimensional algorithm produces good Diophantine approximations for all $\theta \in[0,1]^{d}$. This is so if the Szekeres continuous approximation constant $S_{d}$ is finite.

The second open problem is: For which $d$ does $S_{d}=C_{d}$ ? The evidence of Szekeres $[15,16]$, suggests that an exact bound $S_{d}=C_{d}$ can be hoped for only in the two-dimensional case. Cusick [5] proves a result for a ternary linear form which provides mild support for the possibility that $S_{2}=C_{2}$. However at present we do not even know that $S_{2}$ is finite.

A third open problem concerns analogues of algebraic Diophantine approximation for the multi-dimensional Szekeres map $T_{d}$. This consists of the study of the set of purely periodic points $\mathscr{P}_{d}$ of the maps $T_{d}^{(n)}$. In the one-dimensional case these points are analogues of real quadratic irrationals. We call the spectrum of continuous approximation numbers they determine the Szekeres algebraic spectrum. We define the Szekeres algebraic constant $S_{d}^{*}$ to be the supremum over Szekeres continuous approximation numbers (defined by (A.7)) for purely periodic points. This definition is analogous to the algebraic Diophantine approximation constant $A_{d}$ in the sense that any purely periodic point necessarily has coordinates in an algebraic number field. One has $S_{1}=S_{1}^{*}$ by the results of Section 3 . We propose the conjecture:

CONJECTURE 6.1. $S_{d}=S_{d}^{*}$ for all $d \geq 1$.

This rather speculative conjecture asserts that purely periodic trajectories can approximate (in the continuous approximation measure) extremal trajectories for the continuous approximation measure (A.7). It is known, however, that not all trajectories can be approximated by purely periodic trajectories even in the one-dimensional case; indeed the purely periodic trajectories determine exactly the Lagrange spectrum but do not determine the Markoff spectrum, see Cusick [4]. 


\section{Appendix A: Multi-dimensional Szekeres map}

The multi-dimensional version $T_{d}$ of the Szekeres map has a configuration space $\Delta_{d} \times \Delta_{d}^{*} \times \Gamma_{d}^{*}$, where $\Delta_{d}$ and $\Delta_{d}^{*}$ are each $d$-simplices, regarded as embedded in $\mathbb{R}^{d+1}$ by barycentric coordinates

$$
\Delta_{d}=\left\{\left(x_{0}, x_{1}, \ldots, x_{d}\right): \sum_{i=0}^{d} x_{i}=1 \text { and all } x_{i} \geq 0\right\},
$$

and $\Gamma_{d}^{*}$ is a compact space of 'simplex shapes'.

The space $\Gamma_{d}^{*}$ is a compactification of the set $\Gamma_{d}$ of all $d$-simplices in $\mathbb{R}^{d}$ of volume 1 with barycenter at 0 . A general $d$-simplex in $\mathbb{R}^{d}$ can be mapped to one in $\Gamma_{d}$ by a homothety to make its volume equal to 1 , followed by a translation. The space $\Gamma_{d}$ is not compact, but can be conveniently compactified to get $\Gamma_{d}^{*}$ as explained below in the two-dimensional case. In the one-dimensional case $\Gamma_{1}=\Gamma_{1}^{*}$ is a point, hence it never needs to be mentioned in Sections $2-4$.

We write $(\xi, w, s) \in \Delta_{d} \times \Delta_{d}^{*} \times \Gamma_{d}^{*}$ and let

$$
T_{d}(\xi, \mathbf{w}, \mathbf{s})=\left(\xi^{\prime}, \mathbf{w}^{\prime}, \mathbf{s}^{\prime}\right) .
$$

Here $\xi$ identifies a point inside the simplex of shape $s$, and $w$ is a set of weights attached to the vertices of $s$.

Geometrically the map $T_{d}$ proceeds by splitting the simplex s into two subsimplices, by adding a new vertex along one of its edges. The simplex shape $s$ alone determines the edge that is to be split. The Szekeres splitting rule is to choose the edge of s of greatest length in the sup-norm, that is, if $\mathrm{v}_{0}=\left(v_{01}, \ldots, v_{0 d}\right)$ and $\mathrm{v}_{1}=\left(v_{11}, \ldots, v_{1 d}\right)$ are two vertices of $s$, then the edge length is

$$
\left\|\mathrm{v}_{0}-\mathrm{v}_{1}\right\|_{\text {sup }}:=\max _{1 \leq i \leq d}\left|v_{0 i}-v_{1 i}\right| .
$$

(If $s$ has two maximal-length edges, a suitable tiebreaking rule is used.) The weight vector $w$ is then used to determine the location of the new vertex on the edge to be split in $\mathrm{s}$. If $\mathbf{w}=\left(w_{0}, \ldots, w_{d}\right)$ are the weights corresponding to the vertices $\left(v_{0}, \ldots, v_{d}\right)$ of $s$ and if $\left[v_{0}, v_{1}\right]$ is the edge to be split, the new vertex is

$$
\mathbf{v}^{\prime}=\frac{w_{0}}{w_{0}+w_{1}} \mathbf{v}_{0}+\frac{w_{1}}{w_{0}+w_{1}} \mathbf{v}_{1}
$$

The new vertex splits s into two subsimplices $\hat{s}^{(0)}$ and $\hat{s}^{(1)}$ which are the convex hulls of the new vertex plus all the vertices of $s$ excluding one of the original vertices of the split edge. The new simplex shape $s^{\prime}$ is the simplex containing the point $x_{\xi}$ with barycentric coordinates $\xi$, and $\xi^{\prime}$ are the barycentric coordinates of this point in the new simplex $s^{\prime}$. (If this point $x_{\xi}$ is in both subsimplices $\hat{\mathbf{s}}^{(0)}$ and $\hat{\mathbf{s}}^{(1)}$, then a tiebreaking 
rule is used to decide which of $\hat{s}^{(0)}$ or $\hat{s}^{(1)}$ is taken as $\mathbf{s}^{\prime}$.) The new vertex $v^{\prime}$ is assigned the sum of the weights $w_{0}+w_{1}$ of the original vertices on the split edge. Now the weights assigned to the vertices of $s^{\prime}$ add up to more than 1 , and $w^{\prime}$ are these weights rescaled multiplicatively to add up to 1 . (It is useful to think of the weights $w$ as being in $\mathbb{R}_{+}^{d+1}$, with all $\lambda w$ with $\lambda \in \mathbb{R}^{+}$treated as the same.)

The sup-norm edge lengths (A.3) are preserved under the action of the group of symmetrics of the cube, which has $2^{d} d$ ! elements. We can treat two simplex shapes as being the same under this action, provided that the entries of both $\xi$ and $w$ are permuted simultaneously under this action. This freedom to permute coordinates allows us to define the Szekeres map $T_{d}$ in such a way that the edge $\left[\mathrm{v}_{0}^{\prime}, \mathrm{v}_{1}^{\prime}\right]$ of $\mathrm{s}^{\prime}$ is the longest edge of $\mathrm{s}^{\prime}$ in the norm. $T_{2}$ will be described more explicitly later.

Let $(\xi, w, s)$ denote a configuration. Given a sequence of iterates

$$
\left(\xi^{(n)}, \mathrm{w}^{(n)}, \mathrm{s}^{(n)}\right):=T_{d}^{(n)}\left(\xi^{(0)}, \mathrm{w}^{(0)}, \mathrm{s}^{(0)}\right),
$$

one associates to it a multi-dimensional continued fraction expansion $\left\{\mathrm{P}^{(n)}: n \geq 0\right\}$ with each $\mathrm{P}^{(n)} \in G L(d+1, \mathbb{Z})$, as follows. One defines

$$
\mathrm{P}^{(n)}:=\mathrm{A}^{(n)} \ldots \mathrm{A}^{(1)} \mathrm{A}^{(0)},
$$

where

$$
\mathrm{A}^{(0)}=\left[\begin{array}{ccccc}
1 & 0 & 0 & \cdots & 0 \\
1 & 1 & 0 & & 0 \\
1 & 1 & 1 & & 0 \\
& \vdots & & & \vdots \\
1 & 1 & 1 & \cdots & 1
\end{array}\right]
$$

and each $\mathrm{A}^{(j)} \in G L(d+1, \mathbb{Z})$ indicates how the simplex weights $\mathrm{w}^{(j)}$ were obtained from $w^{(j-1)}$, that is, which two vertices were split and which of these were discarded, in terms of the original numbering of the vertices. For example, if at the $j$ th iteration the edge $\left(v_{i}, v_{k}\right)$ was split and $v_{k}$ was discarded, then $A^{(j)}=I+E_{k, i}$, where $E_{k, i}$ is the matrix having a one in the $(k, i)$-th entry and zeros elsewhere. The matrices $A^{(j)}$ are called the partial quotients of the multi-dimensional continued fraction expansion and $\mathrm{P}^{(j)}$ are the convergents, cf. Szekeres [13], Lagarias [10,11].

The sup-norm Diophantine approximation number attached to vertex $j$ of a configuration $(\xi, w, s)$ is

$$
\gamma_{j, d}(\xi, \mathbf{w}, \mathbf{s}):=\frac{w_{j}^{d+1}}{w_{0} w_{1} \cdots w_{j}} \frac{\left(\left\|\mathbf{b} x_{\xi}-\mathbf{b} v_{j}\right\|_{\text {sup }}\right)^{d}}{\operatorname{Vol}(\mathbf{s})} .
$$

(Vol(s) appears explicitly in (A.5) in order that this expression remains invariant under homotheties $\mathrm{s} \rightarrow \lambda \mathrm{s}$.) In the special case where $\xi^{(0)}=\left(1, \theta_{1}, \ldots, \theta_{d}\right)$ with 
$1 \geq \theta_{1} \geq \theta_{2} \geq \cdots \geq \theta_{d} \geq 0$, where $\mathrm{w}^{(0)}=(1 / n, 1 / n, \ldots, 1 / n)$, and where $\mathrm{s}^{(0)}$ is the shape of the simplex in $\mathbb{R}^{d}$ having vertices at $v_{0}=(0,0, \ldots, 0)$ and

$$
v_{i}=e_{1}+e_{2}+\cdots+e_{i}=(1, \ldots, 1,0, \ldots, 0), \quad(i \text { ones }),
$$

for $1 \leq i \leq d$, then the map $T_{d}$ exactly imitates the action of the multi-dimensional continued fraction algorithm of Szekeres $[13]$ on $\left(\theta_{1}, \ldots, \theta_{d}\right)$. In addition

(A.6) $\gamma_{j, d}\left(\xi^{(n)}, \mathrm{w}^{(n)}, \mathrm{s}^{(n)}\right)=\left(q_{j}^{(n)}\right)^{d+1}\left\|\xi-\left(\frac{p_{i j}^{(n)}}{q_{j}^{(n)}}, \cdots, \frac{p_{d j}^{(n)}}{q_{j}^{(n)}}\right)\right\|_{\text {sup }}^{d}, \quad 0 \leq j \leq d$

is just the sup-norm Diophantine approximation quantity for the $j$ th row of the $n$th approximation matrix

$$
\mathrm{P}^{(n)}=\left[\begin{array}{cccc}
p_{10}^{(n)} & \cdots & p_{d 0}^{(n)} & q_{0}^{(n)} \\
\vdots & & & \vdots \\
p_{1 d}^{(n)} & \cdots & p_{d d}^{(n)} & q_{d}^{(n)}
\end{array}\right]
$$

produced by the Szekeres algorithm. The formula (A.6) is the $d$-dimensional generalization of (2.5).

Now define the Szekeres continuous approximation number by

$$
\Gamma_{d}^{*}(\xi, \mathbf{w}, \mathbf{s})=\liminf _{n \rightarrow \infty} \gamma_{j_{n}, d}\left(\xi^{(n)}, \mathbf{w}^{(n)}, \mathbf{s}^{(n)}\right),
$$

where $j_{n}$ is the new vertex of $\mathrm{s}^{(n)}$, that is, the vertex of $\mathrm{s}^{(n)}$ which is not a vertex of $\mathbf{s}^{(n-1)}$. The Szekeres continuous approximation constant is

$$
S_{d}:=\sup \left\{\Gamma_{d}^{*}(\xi, \mathrm{w}, \mathrm{s}): \operatorname{admissible}(\xi, \mathrm{w}, \mathrm{s})\right\},
$$

where $(\xi, \mathrm{w}, \mathrm{s})$ is admissible if all its iterates under $T_{d}$ are well-defined. (That is, we exclude a measure-zero subset of 'bad' configurations.)

For the case $\left(\xi^{(0)}, \mathbf{w}^{(0)}, \mathbf{s}^{(0)}\right)$ corresponding to $\boldsymbol{\theta}=\left(\theta_{1}, \ldots, \theta_{d}\right) \in \mathbb{R}^{d}$, by (A.6) we have

$$
\text { (A.9) } \quad \Gamma_{d}^{*}\left(\xi^{(0)}, \mathbf{w}^{(0)}, \mathrm{s}^{(0)}\right) \geq C_{d}(\theta) \text {, }
$$

where $C_{d}(\theta)$ is the sup-norm constant for $\theta$, given by

$$
C_{d}(\boldsymbol{\theta})=\liminf _{q \rightarrow \infty}\left(q \min _{p \in \mathbb{Z}^{d}}\|q \boldsymbol{\theta}-\boldsymbol{p}\|_{\text {sup }}^{d}\right) .
$$

Consequently one has
(A.11)
$S_{d} \geq C_{d}$ 
We follow Szekeres [15] to specify an explicit form for the two-dimensional Szekeres map $T_{2}$, as well as a compactified space of normalized configurations $\Gamma_{2}^{*}$. We permute coordinates in the space of configurations $\Gamma_{2}$ of shapes of triangles $\left[v_{0}, v_{1}, v_{2}\right]$ in $\mathbb{R}^{2}$ of area 1 so that $\left[v_{0}, v_{1}\right]$ is the largest edge in the metric \|\|$_{\text {sup. Next we dilate }}$ the triangle so that this edge has length 1 , translate it so as to have one endpoint at 0 , and by suitable symmetries of the cube reduce to the case that

$$
\mathrm{v}_{0}=(0,0), \quad \mathrm{v}_{1}=(1, b), \quad \mathrm{v}_{2}=(x, y),
$$

with $0 \leq b \leq 1,0 \leq x \leq 1, x \leq y \leq 1$; see Figure A.1. We break ties when two edges have length 1 by requiring that if $y=1$ then $b \geq x$ and if $y=1, b=x$ then the point $\xi=\left(\xi_{0}, \xi_{1}, \xi_{2}\right) \in \Delta_{2}$ has $\xi_{1} \leq \xi_{2} ;$ cf. Figure A.1. Szekeres calls this a normalized configuration and $\Gamma_{2}^{*}$ denotes the (three-parameter) set of such configurations.

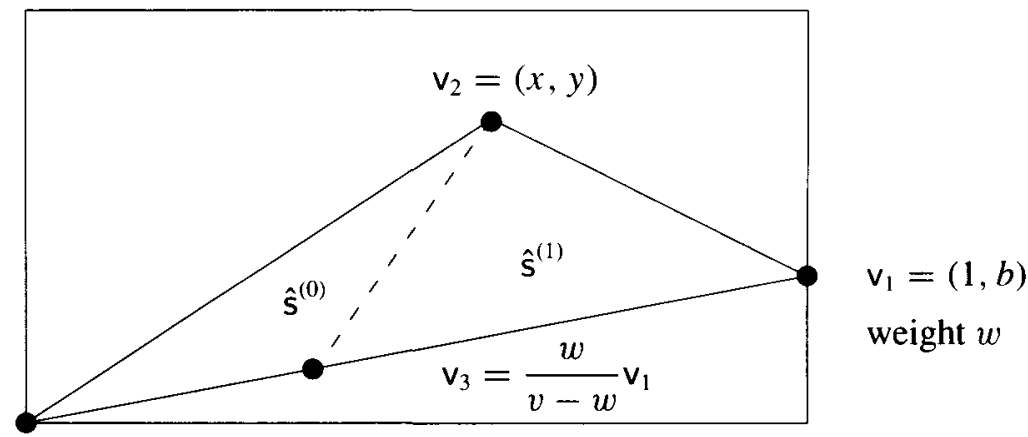

$\mathrm{v}_{0}=(0,0)$

weight $v$

FigurE A.1. A Configuration for the Two-Dimensional Szekeres Map.

With this normalization $\xi=\left(\xi_{1}, \xi_{2}\right)$ determines the interior point $x_{\xi}=\left(\xi_{1} x+\xi_{2}, \xi_{1} y+\right.$ $\left.\xi_{2} b\right)$ in the triangle. Szekeres chooses to scale the weight vector $w=\left(w_{0}, w_{1}, w_{2}\right)$ to have $w_{2}=1$ and to use the parameters $v=w_{0} / w_{2}, w=w_{1} / w_{2}$, so that a configuration is labelled $\left[\xi_{1}, \xi_{2} ; v, w ; b, x, y\right]$ in $\Delta_{2} \times \Delta_{2}^{*} \times \Gamma_{2}^{*}$. The Szekeres map then splits the edge $\left[v_{0}, v_{1}\right]$ at the point

$$
\mathrm{v}_{3}=\frac{v}{v+w} \mathrm{v}_{0}+\frac{w}{v+w} \mathrm{v}_{1} .
$$

Now the triangle $s$ is split into two subtriangles $\hat{s}^{(0)}=\left[v_{0}, v_{2}, v_{3}\right]$ and $\hat{s}^{(1)}=\left[v_{1}, v_{2}, v_{3}\right]$. The point $\xi$ is assigned the triangle it is in, and is assigned $\hat{s}^{(0)}$ if it is on the boundary 
between $\hat{s}^{(0)}$ and $\hat{s}^{(1)}$ (an arbitrary tiebreaking rule). The new configuration $s^{\prime}$ is then normalized, and this defines the map $T_{2}$. Explicit formulae for the map $T_{2}$ involve twelve cases according to which edge of $\mathrm{s}^{\prime}$ is longest, and which vertex of $\mathrm{s}^{\prime}$ is mapped to 0 in the normalization step; we omit these formulae.

The approximation number for the updated point $v_{3}$ with respect to $x_{\xi}$ is:

$$
\gamma=\frac{(v+w)^{2}}{v w} \frac{1}{(y-b x)} \max \left\{\left(\xi_{1} x+\xi_{2}-\frac{w}{v+w}\right)^{2},\left(\xi_{1} y+\xi_{2} b-\frac{b w}{v+w}\right)^{2}\right\} .
$$

\section{Appendix B: Additive map $F$ and additive continued fraction map $\tilde{F}$}

The additive map $F:[0,1]^{2} \rightarrow[0,1]$ is defined by

$$
F(\theta, \Psi)= \begin{cases}\left(\frac{\theta}{1-\theta}, \frac{\Psi}{1+\Psi}\right) & \text { if } 0 \leq \theta \leq 1 / 2, \\ \left(\frac{1-\theta}{\theta}, \frac{1}{1+\Psi}\right) & \text { if } 1 / 2<\theta \leq 1 .\end{cases}
$$

It is invertible almost everywhere, with inverse function

$$
F^{-1}(\theta, \Psi)= \begin{cases}\left(\frac{\theta}{1+\theta}, \frac{\Psi}{1-\Psi}\right) & \text { if } 0 \leq \Psi \leq 1 / 2, \\ \left(\frac{\theta}{1+\theta}, \frac{1-\Psi}{\Psi}\right) & \text { if } 1 / 2<\Psi \leq 1 .\end{cases}
$$

The additive map $F$ is one-to-one and onto when restricted to the domain

$$
\begin{aligned}
\tilde{\mathscr{D}}=\{(\theta, \Psi): & \text { For all } n \in \mathbb{Z}, \text { neither coordinate of } F^{(n)}(\theta, \Psi) \\
& \text { is } 0,1 / 2, \text { or } 1\} .
\end{aligned}
$$

The domain $\tilde{\mathscr{D}}$ has Lebesgue measure one in $[0,1]^{2}$. On this domain $F$ and $F^{-1}$ are completely symmetric, being conjugate to each other by the involution $\sigma(\theta, \Psi)=$ $(\Psi, \theta)$, that is,

$$
F^{-1}=\sigma \circ F \circ \sigma .
$$

The symbolic dynamics of the additive map are particularly simple. Under both forward and backward iteration it is driven by the Farey shift map

$$
S(x)= \begin{cases}\frac{x}{1-x} & \text { if } 0 \leq x \leq 1 / 2 \\ \frac{1-x}{x} & \text { if } 1 / 2<x \leq 1\end{cases}
$$


whose symbolic dynamics are a Bernoulli shift on two symbols.

The additive map $F$ is conjugate to the additive reduction map $U$ of Section 5, by the map

$$
\Psi(\theta, \Psi):=\left(\theta, \frac{\Psi}{1-\Psi}\right)
$$

that is, the following diagram commutes:

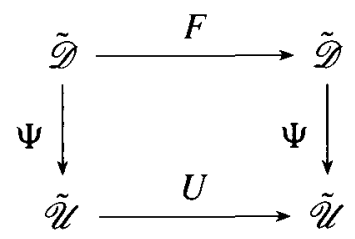

The map $\Psi$ has inverse map

$$
\Psi^{-1}\left(\rho_{1}, \rho_{2}\right):=\left(\rho_{1}, \frac{\rho_{2}}{1+\rho_{2}}\right)
$$

From (B.6) and (5.14) one calculates that the conjugation map $\tilde{\Phi}$ from the Szekeres map $T$ to the additive map is:

$$
(\theta, \psi)=\tilde{\Phi}\left(\xi_{0}, w_{0}\right):=\left(\frac{1}{w_{0}}, \frac{\xi_{0} w_{0}}{1-\xi_{0}}\right)
$$

The map $\tilde{\Psi}$ has inverse map:

$$
(\xi, w)=\tilde{\Phi}^{-1}(\theta, \Psi):=\left(\frac{\theta \psi}{\theta+\psi}, \frac{1}{\theta}\right)
$$

The height function associated to geodesics in $(\theta, \psi)$-coordinates is

$$
h t(\theta, \psi)=\frac{1}{2}\left(\theta+\psi^{-1}\right)
$$

The additive map $F$ is also related in a simple way to the additive contınued fraction map $\tilde{F}(\alpha, \beta, \lambda, \mu)$ constructed in Arnoux and Nogueira [2]. They define the additive continued fraction map $\tilde{F}$ on each domain

$$
\mathscr{D}_{H}=\{(\alpha, \beta, \lambda, \mu): \alpha \lambda+\beta \mu=H, \beta>\alpha>0, \lambda>0, \mu>0\}
$$

with $H>0$ by

(B.10) $\quad \tilde{F}(\alpha, \beta, \lambda, \mu)= \begin{cases}(\alpha, \beta-\alpha, \lambda+\mu, \mu) & \text { if } \alpha \leq \beta-\alpha \\ (\beta-\alpha, \alpha, \mu, \lambda+\mu & \text { if } \alpha>\beta-\alpha .\end{cases}$ 
The quantity $H=\alpha \lambda+\beta \mu$ is invariant under this map; each domain $\mathscr{D}_{H}$ is three dimensional. The map $\tilde{F}$ is one-to-one on $\mathscr{D}_{H}$ off a set of measure zero and has the inverse function

$$
\tilde{F}^{-1}(\alpha, \beta, \lambda, \mu)= \begin{cases}(\alpha, \alpha+\beta, \lambda-\mu, \mu) & \text { if } \lambda \geq \mu \\ (\beta, \alpha+\beta, \lambda, \mu-\lambda) & \text { if } \lambda<\mu\end{cases}
$$

Both $\tilde{F}$ and $\tilde{F}^{-1}$ are defined on

$$
\mathscr{D}=\bigcup\left\{\mathscr{D}_{H}: H>0\right\}=\{(\alpha, \beta, \lambda, \mu): \beta>\alpha>0, \lambda>0, \mu>0\} .
$$

We obtain the two-parameter map $F$ as a quotient of $\tilde{F}$. Observe that $\tilde{F}$ and $\tilde{F}^{-1}$ are both homogeneous in $(\alpha, \beta)$ and in $(\lambda, \mu)$. Consequently the point $(\alpha, \beta, \lambda, \mu)$ and any point $\left(t_{1} \alpha, t_{1} \beta, t_{2} \lambda, t_{2} \mu\right)$ for $t_{1}>0, t_{2}>0$ have exactly the same symbolic dynamics when iterated forwards under $\tilde{F}$ and when iterated backwards under $\tilde{F}^{-1}$. So we may rescale $t_{1}$ and $t_{2}$ at each step without affecting the symbolic dynamics. The map $\tilde{F}$ on $\mathscr{D}$ thus induces a well-defined quotient action on two-dimensional pencils

$$
[(\alpha, \beta, \lambda, \mu)]=\left\{\left(t_{1} \alpha, t_{1} \beta, t_{2} \lambda, t_{2} \mu\right): t_{1}>0, t_{2}>0\right\} .
$$

We choose a canonical representative in $[(\alpha, \beta, \lambda, \mu)]$ by taking $t_{1}=1 / \beta$ and $t_{2}=$ $1 /(\lambda+\mu)$ to get $(\alpha / \beta, 1, \lambda /(\lambda+\mu), \mu /(\lambda+\mu))$. There are then only two free parameters $\theta=\alpha / \beta$ and $\psi=\mu /(\lambda+\mu)$, and this point is $(\theta, 1,1-\psi, \psi)$. The induced quotient action of $\tilde{F}$ on the first and last coordinate yields the additive map

$$
F(\theta, \psi)= \begin{cases}\left(\frac{\theta}{1-\theta}, \frac{\psi}{1+\psi}\right) & \text { if } 0 \leq \theta \leq 1 / 2, \\ \left(\frac{1-\theta}{\theta}, \frac{1}{1+\psi}\right) & \text { if } 1 / 2 \leq \theta \leq 1 .\end{cases}
$$

This quotient action of $\tilde{F}$ yielding $F$ is unrelated to the geometric interpretation of $\tilde{F}$ given in Arnoux and Nogueira [2, §4]. Their geometric interpretation depends on the invariance of the quantity $H$ (that is, the $2 \times 2$ matrix $\left(\begin{array}{cc}\alpha & \beta \\ -\lambda & \mu\end{array}\right)$ has determinant $H$ ), which is not preserved in the identification of the quotient map on pencils (B.12).

\section{References}

[1] W. W. Adams, 'The best two-dimensional Diophantine approximation constant for cubic irrationals', Pacific J. Math. 91 (1980), 29-30.

[2] P. Amoux and A. Nogueira, 'Mesures de Gauss pour des algorithmes de fractions continues multidimensionales', Ann. Sci. École Norm. Sup. (to appear). 
[3] J. W. S. Cassels, 'Simultaneous Diophantine approximation', J. London Math. Soc. 30 (1955), $119-121$.

[4] T. W. Cusick, 'The connection between the Lagrange and Markoff spectra', Duke Math. J. 42 (1975), 507-517.

[5] —_ 'The Szekeres multidimensional continued fraction', Math. Comp. 31 (1977), 280-317.

[6] T. W. Cusick and M. Flahive, The Markoff and Lagrange spectra (Amer. Math. Soc., Providence, 1991).

[7] T. W. Cusick and S. Krass, 'Formulas for some Diophantine approximation constants', J. Australian Math. Soc. (Series A) 44 (1988), 311-323.

[8] L. E. Dickson, Modern elementary theory of numbers (Univ. of Chicago Press, Chicago, 1939).

[9] S. Krass, 'Estimates for $n$-dimensional Diophantine approximation constants for $n \geq 4$ ', J. Number Theory 20 (1985), 172-176.

[10] J. C. Lagarias, 'The quality of the Diophantine approximations produced by the Jacobi-Perron algorithm and related algorithms', Monatsh. Math. 115 (1993), 299-328.

[11] _ ' 'Geodesic multidimensional continued fractions', Proc. London Math. Soc. (1994) (to appear).

[12] I. Richards, 'Continued fractions without tears', Math. Mag. 54 (1981), 163-171.

[13] G. Szekeres, 'Multidimensional continued fractions', Annalés Sci. Budapest Eötvos Sect. Math. 13 (1970), 113-140.

[14] - 'The $n$-dimensional approximation constant', Bull. Austral. Math. Soc. 29(1984), 119-125.

[15] - 'Computer examination of the 2-dimensional simultaneous approximation constant', Ars. Combin. 19A (1985), 237-243.

[16] - 'Search for the three dimensional approximation constant', in: Diophantine analysis (eds. J. Loxton and A. van der Poorten), London Math. Soc. Lecture Notes 106, (Cambridge U. Press, Cambridge, 1986) pp. 139-146.

AT\&T Bell Laboratories

Murray Hill, New Jersey 07974

USA

e-mail: jcl@research.att.com
Brigham Young University Provo, Utah 84602

USA

e-mail: andy@hamblin.math.byu.edu 